\title{
Métodos diagnósticos en hipertensión portal
}

\section{Diagnostic methods of portal hypertension}

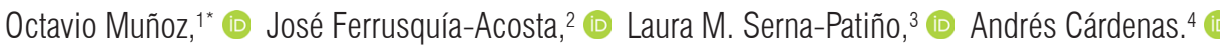

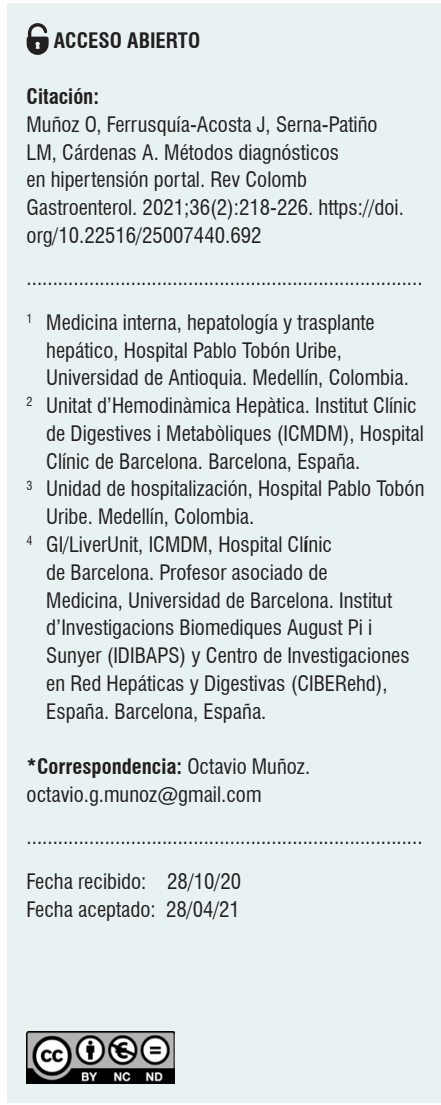

\begin{abstract}
Resumen
La hipertensión portal es un síndrome caracterizado por el incremento en el gradiente de presión portal, definido por la diferencia entre la presión de la porta y la vena cava inferior. Esta presión depende del flujo venoso y la resistencia vascular. En los pacientes cirróticos estas dos variables están alteradas de manera suprafisiológica, inicialmente por la lesión estructural dependiente de fibrosis y los nódulos de regeneración y posteriormente por cambios dinámicos vasculares que causan vasoconstricción intrahepática y vasodilatación esplácnica, lo cual explica las manifestaciones sistémicas de la cirrosis. La importancia de la hipertensión portal radica en la frecuencia y severidad de las complicaciones asociadas, especialmente la hemorragia variceal y otras como ascitis, peritonitis bacteriana espontanea, síndrome hepatorrenal y encefalopatía hepática. El objetivo de este artículo es realizar una revisión actualizada sobre el uso de las pruebas diagnósticas invasivas y no invasivas disponibles para el estudio de la hipertensión portal y su aplicación en la práctica clínica.
\end{abstract}

\section{Palabras clave \\ Hipertensión portal, sistema porta, cirrosis hepática.}

\section{Abstract}

Portal hypertension is characterized by an increase in the portal pressure gradient, which is defined as the difference between the portal venous pressure and the pressure within the inferior vena cava. Such a pressure depends on venous flow and vascular resistance. In patients with cirrhosis, both variables are altered, initially due to fibrosis-dependent structural injury and regeneration nodules, and subsequently by vascular dynamic changes that cause intrahepatic vasoconstriction and splanchnic vasodilation, which explains the systemic manifestations of cirrhosis. The importance of portal hypertension lies in the frequency and severity of associated complications, especially variceal hemorrhage, but also ascites, spontaneous bacterial peritonitis, hepatorenal syndrome, and hepatic encephalopathy. The objective of this article is to carry out an updated review on the use of invasive and non-invasive diagnostic tests available for the study of portal hypertension and their application in clinical practice.

\section{Keywords}

Portal hypertension; Portal system; Hepatic cirrosis.

\section{INTRODUCCIÓN}

La hipertensión portal es un síndrome caracterizado por el incremento en el gradiente de presión portal (PPG), definido por la diferencia de presión entre la vena porta y la vena cava inferior. Los valores normales del PGG varían entre 1 y $5 \mathrm{~mm} \mathrm{Hg}$, mientras que los valores $\geq 6 \mathrm{~mm} \mathrm{Hg}$ indican la presencia de hipertensión portal $(1,2)$. La cirro- 
sis es la causa más frecuente de hipertensión portal y es responsable del $90 \%$ de los casos en Estados Unidos y Europa. Menos del $10 \%$ de los casos corresponde a causas de origen no cirrótico. Dentro de las patologías representativas de este grupo se encuentran las enfermedades vasculares como trombosis portal, síndrome de Budd-Chiari, enfermedad vascular porto-sinusoidal, insuficiencia cardíaca derecha, esquistosomiasis, entre otras (3).

La medición del gradiente de presión venosa hepática (GPVH) es considerado el estándar de oro para el diagnóstico (4). Sin embargo, la presencia de manifestaciones clínicas en el escenario de pacientes con factores de riesgo podría ser suficiente para hacer el diagnóstico (5). La hipertensión portal será clínicamente significativa cuando el GPVH sobrepase el nivel crítico de los $10 \mathrm{~mm} \mathrm{Hg}$; en este punto ocurren cambios en los lechos vasculares extrahepáticos que llevan a la formación de colaterales portosistémicas y a la vasodilatación esplácnica, que contribuyen al incremento progresivo de la presión portal y, finalmente, a su expresión clínica (desarrollo de várices esofágicas, ascitis, encefalopatía y hepatocarcinoma) (6), y es silente en los estadios iniciales, en los que la GPVH aún se encuentra en el rango de 6-9 $\mathrm{mm} \mathrm{Hg}$.

La relevancia de la hipertensión portal radica en la frecuencia y gravedad de las complicaciones asociadas. Su principal forma de presentación es la hemorragia varicosa (7), que incluye también entidades como la gastropatía hipertensiva, peritonitis bacteriana espontánea, síndrome hepatorrenal y hepatopulmonar, encefalopatía hepática, entre otras; todas ellas asociadas con el aumento en la mortalidad y la necesidad de trasplante hepático en pacientes con cirrosis (8). La gravedad de la hipertensión portal se relaciona directamente con la probabilidad de desarrollar este tipo de complicaciones y es un indicador pronóstico independiente (9). Por ejemplo, un GPVH $>12 \mathrm{~mm} \mathrm{Hg}$ aumenta el riesgo de sangrado por várices esofágicas. Se ha observado un incremento de la mortalidad cuando existe un aumento en el GVPH por encima de $16 \mathrm{~mm} \mathrm{Hg}$ y en los sangrados varicosos, un GVPH $>20 \mathrm{~mm} \mathrm{Hg}$ predice la posibilidad de fallar en el control del sangrado y disminuye la supervivencia a 1 año (Figura 1) $(1,10)$. Cuando la cirrosis es diagnosticada, las várices esofágicas se presentan en el $40 \%$ de los pacientes compensados y en el $60 \%$ de los pacientes con ascitis; adicionalmente, hasta un $30 \%$ a $50 \%$ de los pacientes con sangrado agudo pueden morir en las siguientes 6 semanas $(11,12)$. Por ende, lograr reducciones en la presión portal disminuye la frecuencia de descompensación y mejora la supervivencia.

\section{FISIOPATOLOGÍA DE LA HIPERTENSIÓN PORTAL}

La presión portal, al igual que en cualquier otro lecho vascular, es el producto del flujo venoso por la resistencia vascular; de hecho, el desarrollo de la hipertensión portal en las enfermedades hepáticas crónicas es el resultado de la elevación suprafisiológica de ambos parámetros (13).

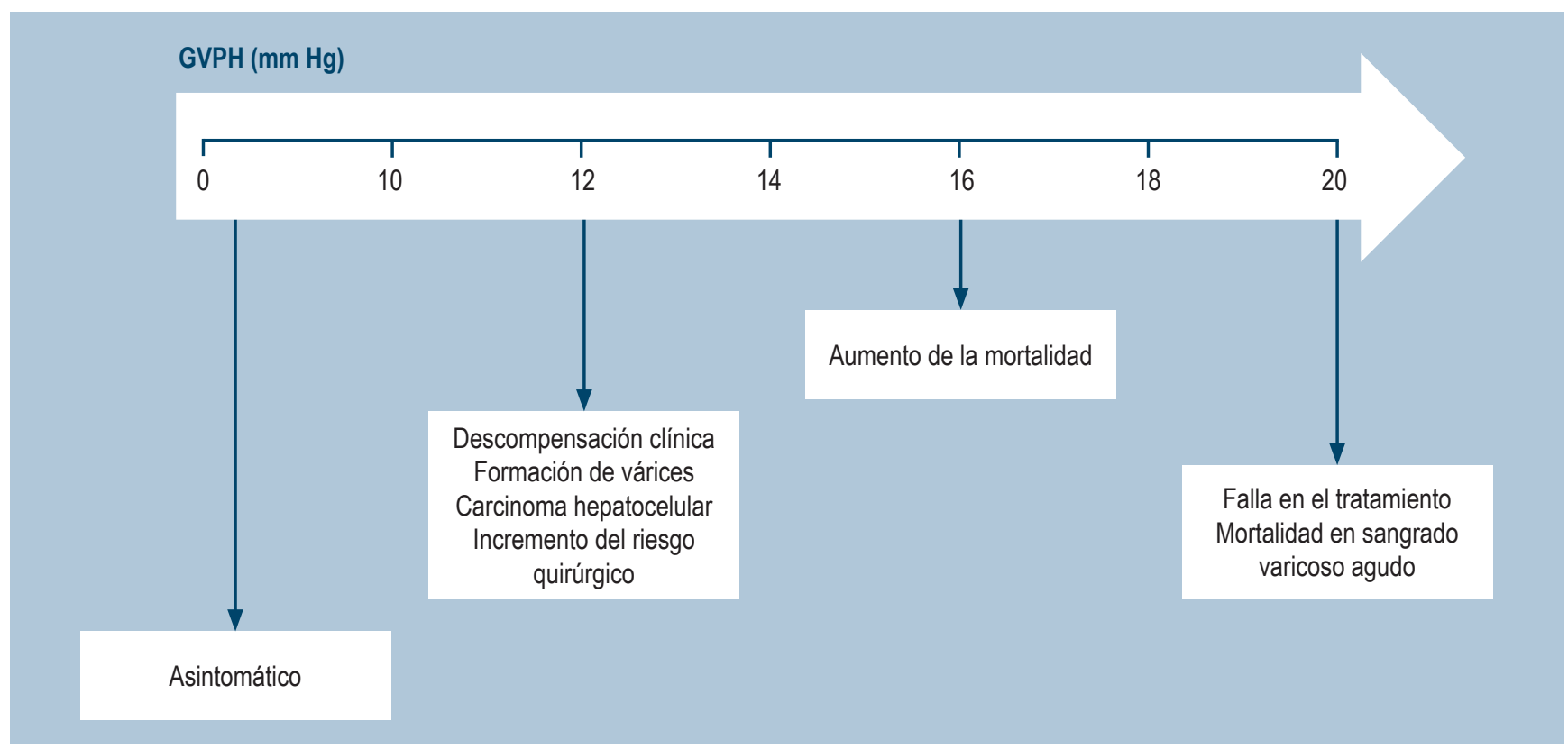

Figura 1. Riesgos asociados con el incremento de los gradientes de presión venosa hepática. 
Incremento en el flujo portal y circulación hiperdinámica

Los pacientes con hipertensión portal presentan un fenómeno de vasodilatación periférica, dependiente de un desequilibrio en la síntesis de vasodilatadores por la disfunción endotelial en la microvasculatura hepática como las prostaglandinas, el óxido nítrico y el glucagón, y un incremento en la producción de vasoconstrictores (endotelina, norepinefrina, tromboxano $A_{2}$, angiotensina II), todo lo cual está relacionado con el componente dinámico de la resistencia hepática $(14,15)$. El aumento de la presión portal envía señales al sistema esplácnico para promover la vasodilatación e incrementar el flujo portal (el óxido nítrico es reconocido como uno de los principales mediadores de la vasodilatación esplácnica y angiogénesis). Otra consecuencia de la vasodilatación esplácnica es la derivación del gasto cardíaco desde la circulación sistémica hacia el mesenterio, lo que produce hipotensión sistémica e hipoperfusión renal relativa (16). Este decremento en el volumen arterial central lleva a la activación de mecanismos compensadores como el sistema renina-angiotensina-aldosterona, el sistema nervioso simpático y la vasopresina, que conducen a la retención de sodio y agua, lo que aumenta el volumen plasmático (17). La circulación hiperdinámica impulsada por la activación del sistema adrenérgico $\beta$ es otra forma de respuesta compensatoria a la hipotensión sistémica.

\section{Incremento en la resistencia vascular}

El hígado es un sistema de baja resistencia y su microcirculación es peculiar, ya que la relación de la resistencia pre- y postsinusoidal es de 49:1, en contraste con otros tejidos como el músculo esquelético, cuya relación es de 4:1; esta característica es un mecanismo protector para el endotelio hepático que es discontinuo (17). Los sitios de incremento en la resistencia pueden ser prehepáticos, hepáticos o poshepáticos, y de esta manera se han clasificado los síndromes de hipertensión portal. Tradicionalmente, estos fenómenos se han explicado por compromiso mecánico del flujo venoso portal y distorsión de la anatomía vascular, dados por trombosis, fibrosis y nódulos.

Estos mecanismos explican, en parte, el compromiso prey poshepático; pero en el intrahepático, y especialmente en el caso de la cirrosis, las células endoteliales sinusoidales se vuelven disfuncionales, por lo que adquieren un fenotipo vasoconstrictor. Este desequilibrio resultante promueve la contracción de las células estrelladas hepáticas, miofibroblastos y células de músculo liso vascular, lo que genera un aumento del tono vascular hepático y de la presión portal (8). Como consecuencia de la activación de las células estrelladas, existe una profunda alteración de la estructura sinusoidal, caracterizada por la pérdida de sus fenestraciones, que deteriora la dispersión natural de las fuerzas hidrostáticas en los sinusoides normales (18).

En resumen, la formación de tejido cicatricial y nódulos regenerativos que ocurre en el paciente cirrótico (cambios estructurales) conduce a un aumento de la resistencia vascular intrahepática y, en consecuencia, de la presión portal. Estos cambios estructurales pueden observarse en las primeras etapas de la hipertensión portal relacionada con la cirrosis, seguidos de vasodilatación esplácnica compensadora, que a su vez resulta en un aumento del flujo sanguíneo portal, lo que empeora aún más la presión portal (Figura 2) $(19,20)$.

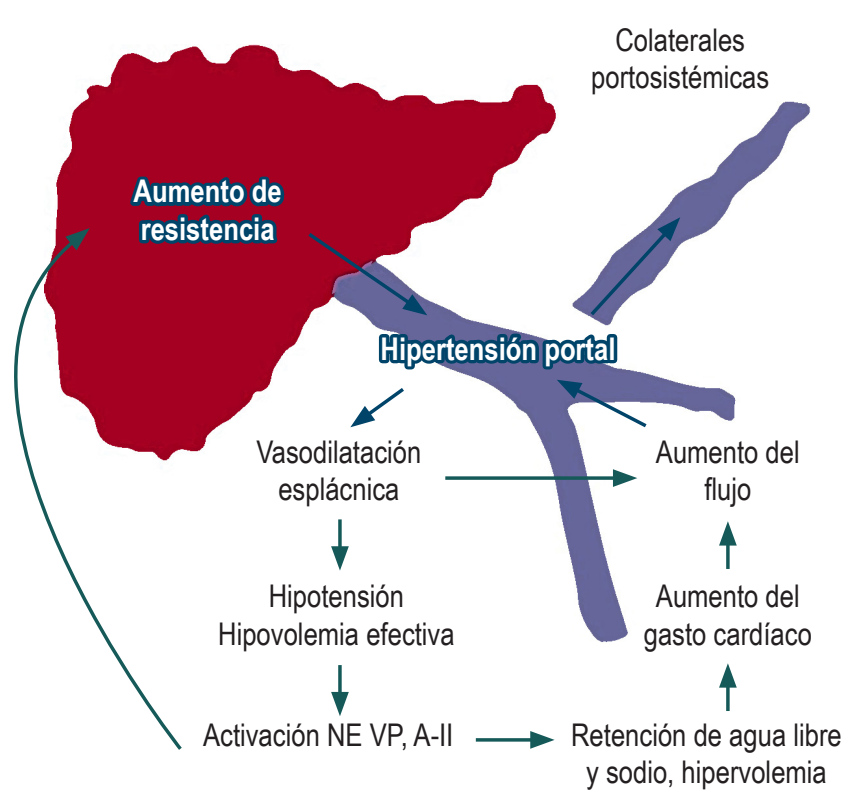

Figura 2. Fisiopatología de la hipertensión portal. AII: Angiotentesina; NE: Norepinefrina; VP: Vasopresina.

\section{DIAGNÓSTICO DE LA HIPERTENSIÓN PORTAL}

Aunque el diagnóstico definitivo de la hipertensión portal requiere el uso de métodos invasivos, un diagnóstico preciso podría realizarse con base en la presencia de complicaciones asociadas con la hipertensión portal y la exclusión de otras causas potenciales. Debido a que la cirrosis representa la principal causa de hipertensión portal, rara vez se necesita una evaluación invasiva de la presión portal en la práctica clínica convencional con fines diagnósticos. Sin embargo, las mediciones invasivas son importantes en la evaluación de la gravedad de la hipertensión portal, con fines pronósticos o cuando existen dudas en el diagnóstico inicial (21).

Los pacientes con hipertensión portal se presentan comúnmente con signos y síntomas generales de las enfermedades hepáticas crónicas, manifestadas por ictericia, ascitis, nevus 
aracnoideos, eritema palmar, atrofia testicular, ginecomastia, hipertrofia de parótidas y atrofia muscular. Adicionalmente, pueden presentar signos de encefalopatía hepática que pueden ser tan sutiles como la falta de concentración e irritabilidad, o tan graves como el estupor o el coma.

El sistema portal tiene colaterales que lo intercomunican con la circulación sistémica en un intento por descomprimirlo. Estos sistemas venosos colaterales hacen parte del síndrome de hipertensión portal. Las colaterales de mayor importancia clínica son las dilataciones venosas esofágicas y gástricas, ya que pueden sangrar de forma aguda (22). La vena umbilical, originada en la vena porta izquierda, puede dilatarse por causas intrahepáticas que se manifiestan por dilataciones venosas en la pared abdominal anterior. A nivel rectal, los sistemas venosos de derivación colateral de la vena mesentérica inferior se manifiestan como várices rectales o dilataciones hemorroidales, que son poco específicas. La circulación colateral retroperitoneal no puede ser clínicamente evaluada y la presencia de dilataciones venosas dorsales es extraordinaria, y se asocia con mayor frecuencia a fenómenos obstructivos de la vena cava inferior. Aunque la esplenomegalia está comúnmente presente en los síndromes de hipertensión portal, no existe una relación de proporcionalidad entre el tamaño del bazo y la presión en el sistema portal (20).

\section{MÉTODOS INVASIVOS PARA EVALUAR LA HIPERTENSIÓN PORTAL}

\section{Gradiente de presión venosa hepática}

Por una parte, el gradiente de presión venoso hepático ha demostrado ser una técnica segura, precisa y reproducible para estimar de forma cuantitativa la presión portal, además de ser útil para valorar la respuesta a la terapia farmacológica, y puede tener un significado pronóstico en el paciente con cirrosis. Debido a su escasa disponibilidad, el uso rutinario en la práctica clínica está limitado a centros especializados. El procedimiento consiste en la inserción de un catéter a través de la vena yugular interna, vena cubital o femoral guiado por fluoroscopia, el cual se avanza hacia una de las venas suprahepáticas. Una vez se alcanza la posición, se infla el balón para ocluir la vena y medir la presión venosa hepática en cuña (WHVP). Por otra parte, la presión venosa hepática libre (FHVP) se mide a una distancia de $2-3 \mathrm{~cm}$ del orificio de las venas hepáticas. La diferencia entre esas 2 presiones es igual al GPVH (23). El valor normal del GPVH fluctúa entre $2 \mathrm{a} 5 \mathrm{~mm} \mathrm{Hg}$ y por encima de $5 \mathrm{~mm} \mathrm{Hg}$ se define como hipertensión portal. La hipertensión portal clínicamente significativa se diagnóstica cuando el GPVH es $\geq 10 \mathrm{~mm} \mathrm{Hg}$. En pacientes con hipertensión portal de causa desconocida, el hallazgo de un aumento del GPVH debido a un aumento en la WHVP indica una elevación de la presión sinusoidal, que con mayor frecuencia está asociada con cirrosis. Un valor normal del GVPH, con una WHVP y FHVP normal, es típica de la hipertensión portal presinusoidal, mientras que en la hipertensión portal poshepática existirá un incremento de la FHVP y WHVP (22).

La reducción farmacológica o mecánica del GVPH con la utilización de derivación intrahepática transyugular portosistémica (TIPS) reduce el desarrollo de descompensación y mejora la supervivencia (24). Los medicamentos vasoactivos, especialmente los bloqueantes $\beta$ no selectivos y los nitratos, disminuyen el GPVH en pacientes cirróticos; una buena respuesta hemodinámica se define como una reducción del GPVH $<12 \mathrm{~mm} \mathrm{Hg}$ o un descenso al menos del $20 \%$ del valor basal, que indica una mejoría con el tratamiento de la enfermedad hepática (25). Cuando se alcanza la meta de reducción, el riesgo de sangrado varicoso, ascitis, peritonitis bacteriana espontánea o síndrome hepatorrenal se disminuye dramáticamente, y esto mejora la supervivencia. La falla en alcanzar estas metas de reducción es el predictor independiente más fuerte de sangrado varicoso o resangrado $(24,26)$.

A pesar de ser considerado un procedimiento invasivo, se trata de una técnica muy segura y bien tolerada por los pacientes. La mayoría de las complicaciones ocurren en relación con la punción venosa y, por tanto, se limitan al sitio de punción (dolor, hematoma, seroma).

\section{Evaluación endoscópica}

La endoscopia digestiva superior es una herramienta importante en la evaluación de la hipertensión portal y clásicamente se ha recomendado a todos los pacientes con cirrosis como tamizaje para documentar la presencia de várices (27). Identificar y tratar a los pacientes con várices de alto riesgo mejora los desenlaces clínicos. Se estima que el $30 \%$ de los pacientes con cirrosis compensada y $60 \%$ de los pacientes con cirrosis descompensada tienen várices al momento del diagnóstico. Cada episodio de hemorragia varicosa está asociada con un aumento en el $20 \%$ de la mortalidad, además de un riesgo del $70 \%$ de desarrollar recurrencia del sangrado en el año posterior al episodio inicial (3).

La endoscopia ofrece otras ventajas: evalúa la presencia y las características de las várices gastroesofágicas, las clasifica por tamaño, graduadas en pequeñas $(<5 \mathrm{~mm})$ o grandes (> $5 \mathrm{~mm}$ ), e informa sobre la apariencia de la pared de las várices (signos rojos en la pared varicosa y manchas rojo cereza) que, junto con la clasificación de Child-Pugh, constituyen también un índice pronóstico de sangrado (Figura 3). Uno de los sistemas más completos es el japonés, que evalúa 6 características: localización, forma/tamaño, color, 


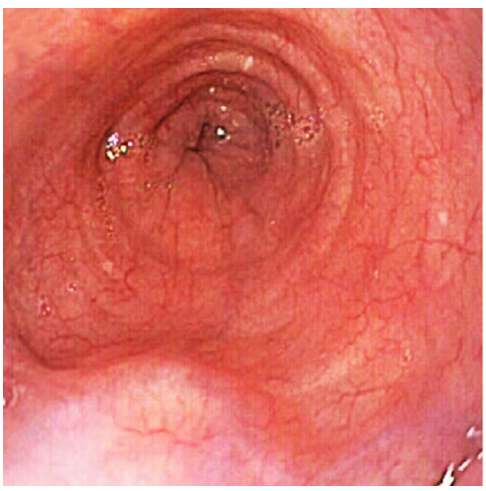

Sin várices

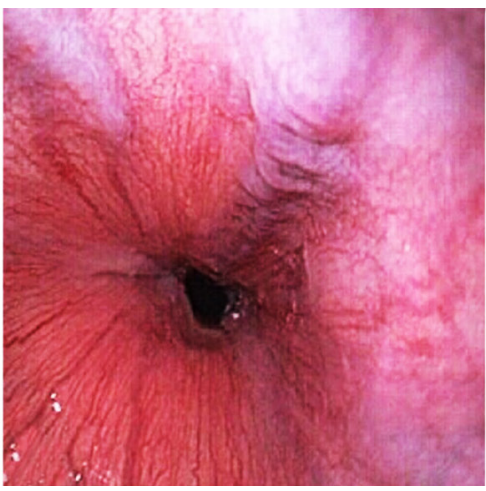

Várices pequeñas

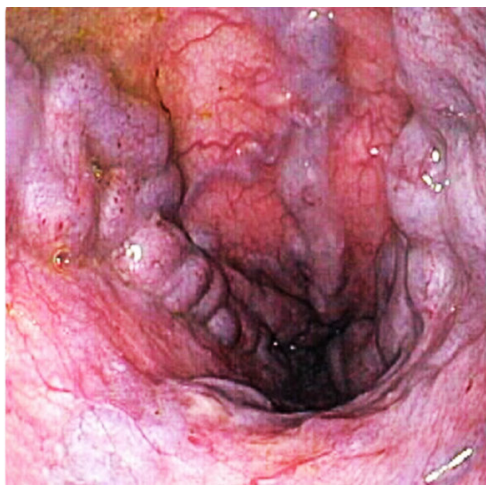

Várices grandes

Figura 3. Clasificación de várices esofágicas.

signos rojos, evidencia de sangrado y características de la mucosa (27). Los cambios endoscópicos no solo se limitan a nivel esofágico, también es común encontrar cambios vasculopáticos a nivel gástrico, dados por la presencia de várices gástricas y gastropatía hipertensiva (28). Las várices gástricas son menos prevalentes que las esofágicas y son la etiología del $5 \%-10 \%$ de casos de sangrado digestivo en pacientes cirróticos. La gastropatía portal hipertensiva es otro hallazgo común en los pacientes con cirrosis, con prevalencias desde el $11 \%$ hasta el $80 \%$, y la ectopia vascular antral gástrica puede ocurrir en el $30 \%$ de los pacientes con hipertensión portal $(1,27)$. La mucosa colónica también presenta cambios vasculares en los pacientes con hipertensión portal, caracterizados por hemorroides, várices anorrectales y colopatía dada por capilares tortuosos e irregulares asociados con edema de la lámina propia y signos inflamatorios crónicos. Adicionalmente, con la utilización de la cápsula endoscópica se han logrado precisar alteraciones vasculares en el intestino delgado, que definen el término de enteropatía hipertensiva portal (29).

\section{Ultrasonografía endoscópica (USE)}

La USE permite detectar tempranamente los cambios de la circulación colateral en los pacientes con hipertensión portal. La USE permite no solo evaluar el drenaje intrínseco del esófago que se presenta como várices visibles en la endoscopia tradicional, sino también la identificación de venas extrínsecas a lo largo de la unión gastroesofágica, incluidas las várices periesofágicas y las paraesofágicas (30). Entonces, este método permite la visualización de várices esofágicas, gástricas, colaterales venosas, el sistema portal, la vena ácigos y el conducto torácico (31, 32). Adicionalmente, este sistema permite valorar las venas esofágicas perforantes y ha demostrado que los pacientes con várices paraesofágicas grandes tienen mayor riesgo de recurrencia y sangrado después de la terapia (33).

La ventaja de la USE sobre la endoscopia tradicional es la habilidad de identificar várices esofágicas con mayor riesgo de sangrado. La endosonografía permite medir el tamaño de las várices y visualizar manchas rojas que pueden aparecer como proyecciones saculares similares a un aneurisma en la superficie de la várice, lo que representa una debilidad focal de la pared, con mayor riesgo de ruptura. Los estudios han demostrado la precisión de la USE para determinar el radio de las várices y el espesor de la pared, lo que puede ser útil como una forma mínimamente invasiva de predecir la presión intravaricosa. La tensión de la pared varicosa es directamente proporcional a la presión y al radio de las várices e inversamente proporcional al grosor de su pared, y el riesgo de ruptura está directamente relacionado con la tensión de la pared. A pesar de todas estas ventajas, su uso en la práctica clínica no es común (31).

\section{MÉTODOS NO INVASIVOS PARA EVALUAR LA HIPERTENSIÓN PORTAL}

Los riesgos derivados de los procedimientos invasivos, el uso de sedación o anestesia y la posibilidad de efectos adversos o complicaciones ha creado la necesidad de investigar el uso de pruebas no invasivas para el estudio de la hipertensión portal.

\section{Marcadores serológicos y exámenes de laboratorio}

El recuento de plaquetas es usado en todo el mundo como signo indirecto de hipertensión portal. La trombocitopenia moderada se presenta en $10 \%-15 \%$ de los pacientes con 
cirrosis hepática, un recuento $<150000$ células tiene un valor predictivo positivo para identificar várices esofágicas medianas y grandes de solo $15,1 \%$ (34). Además, los cambios en el recuento de plaquetas en los pacientes con hepatopatía crónica no tienen una relación directa con el gradiente de presión venoso hepático y se ve afectado por el secuestro esplénico, la supresión medular y la disminución en los niveles de trombopoyetina (35). Cuando se usa la relación entre el recuento de plaquetas y el diámetro esplénico con un punto de corte $<909$, se mejora el desempeño para descartar la presencia de hipertensión portal significativa (36). Exámenes de laboratorio como la albúmina, bilirrubina, índice internacional normalizado (INR) o su combinación en la puntuación de Child-Pugh se correlacionan con el GPVH y con la prevalencia y el grado de las várices esofágicas en pacientes con cirrosis (1).

Hay otros marcadores indirectos de la fibrosis hepática que se han relacionado con el grado de hipertensión portal. Entre ellos están la relación alanina-aminotransferasa/ aspartato-aminotransferasa (ALT/AST), AST/plaquetas, índice FIB-4 y los puntajes de Forns y de Lok. A pesar de su utilidad para evaluar la fibrosis, su desempeño diagnostico en el caso de hipertensión portal es bajo (37). Más recientemente se han tratado de usar otros biomarcadores directos como la laminina, el ácido hialurónico, el procolágeno tipo III, el factor de Von Willebrand y el antígeno de diferenciación (CD) 163 soluble, pero los resultados son heterogéneos y su utilidad clínica permanece incierta (38).

\section{Ecografía abdominal}

Muchos investigadores han hecho esfuerzos para diagnosticar la hipertensión portal utilizando sistemas de ultrasonografía Doppler; el ultrasonido es seguro, económico y permite valorar signos indirectos de enfermedad hepática. Los estudios de ecografía Doppler son útiles para evaluar la permeabilidad de la vena porta, determinar la dirección y medir el flujo sanguíneo a través de los lechos portal y esplácnico, además de permitir la visualización de anormalidades morfológicas asociadas con la hipertensión portal como la dilatación del sistema venoso portal, esplenomegalia y presencia de colaterales portosistémicas (39). Entre los parámetros hemodinámicos que han sido estudiados están los diámetros y la velocidad del flujo esplácnico, la variación de los calibres vasculares con la respiración, el índice de congestión venoso portal, el índice de pulso y la resistencia de las arterias del sistema portal (27). Como en otras áreas de la medicina diagnóstica, la USE cuenta con el problema de la subjetividad por ser una prueba dependiente del operador; adicionalmente, la precisión diagnóstica depende de factores técnicos y la variabilidad intraobservador e interobservador es alta (40).

\section{Tomografía axial computarizada (TAC) y resonancia magnética (RM)}

Se ha demostrado que la arquitectura hepática, su perfusión y el flujo sanguíneo en la arteria esplénica determinada por $\mathrm{RM}$ se correlacionan con la presión portal. En un estudio realizado en pacientes con cirrosis, se encontró que el tiempo de relajación longitudinal hepático y la velocidad de la arteria esplénica se correlacionaban significativamente con el GPVH (4). Con estos métodos, la vasculatura portal puede ser visualizada y valorada de una manera cualitativa con mucha certeza. La mayor definición de las imágenes, las técnicas de reconstrucción tridimensional y los tomógrafos multidetector han permitido la evaluación más precisa del sistema portal y de sus colaterales para poder definir su permeabilidad y los signos de hipertensión portal. En la TAC, los vasos colaterales y las várices son comúnmente vistas en el esófago, el ligamento gastrohepático y el hilio esplénico; adicionalmente, la vena umbilical se puede apreciar recanalizada (41).

La resonancia nuclear magnética permite la cuantificación del flujo de la vena porta y de la vena ácigos como signos indirectos para la valoración de la hipertensión portal $y$, al igual que en la TAC, los hallazgos iniciales están dados por la dilatación de la vena porta y, posteriormente, por la definición de colaterales portosistémicas (42). A pesar de que estas técnicas de imagen permiten identificar signos específicos de hipertensión portal (circulación colateral), no evalúan de forma cuantitativa la hipertensión portal y, por tanto, no son capaces de evaluar sus cambios dinámicos.

La utilidad de estas 2 técnicas de imagen es representativa para pacientes que requieran una valoración detallada del sistema venoso portal, como en la evaluación de la extensión de la trombosis, detección de colangiopatía portal en pacientes con cavernomas portales, mapear la circulación colateral en pacientes con hemorragia varicosa ectópica o para la colocación de TIPS en pacientes complejos, especialmente aquellos con síndrome de Budd-Chiari.

\section{Elastografía}

En los últimos años, la evaluación de la enfermedad hepática ha mejorado sustancialmente tras la introducción de la elastografía. La posibilidad de estimar la fibrosis hepática e indirectamente la gravedad de la hipertensión portal con la medición de la rigidez hepática ha cambiado el tratamiento del paciente (43). El fundamento que llevó al uso de la medición de la rigidez hepática como expresión de la hipertensión portal se basa en el hecho de que la rigidez hepática depende de la cantidad de colágeno y, por tanto, del componente estructural de la presión portal. La mejor correlación entre el GPVH y la medida de rigidez hepática ocurre cuando los valores del 
GPVH se encuentran entre 5 y $12 \mathrm{~mm} \mathrm{Hg}$. Sin embargo, a medida que la hipertensión portal es más grave, la correlación entre la rigidez hepática y el GPVH se pierde; en estos casos, la medición de la rigidez esplénica parece ser un marcador más confiable de hipertensión portal, así como un predictor de descompensación hepática (4). El consenso de Baveno VI sugiere que un valor de fibroscan de 20-25 kPa, solo o en combinación con el recuento de plaquetas y el tamaño del bazo, es suficiente para confirmar la presencia de hipertensión portal significativa en pacientes con cirrosis; además, recomienda que en los pacientes con menos de $20 \mathrm{kPa}$ y más de 150000 plaquetas se puede posponer la realización de la endoscopia de tamizaje, debido a la baja probabilidad de tener várices esofágicas de alto riesgo (44). Con estos criterios, el $21 \%$ de las endoscopias digestivas pueden evitarse y solo se perderían menos del $5 \%$ de los pacientes con várices esofágicas de alto riesgo. En la validación de estos criterios se ha demostrado que se puede clasificar de forma errónea la gravedad de la hipertensión portal en el $2 \%$ de la población (45). También se ha usado la elastografía esplénica, aunque la hipertensión portal tiene relación con la dureza esplénica, cuando el gradiente de presión venosa portal es $>12 \mathrm{~mm} \mathrm{Hg}$, no tiene relación directa con grado de $\mathrm{kPa}$ reportados y, por tanto, su uso clínico para predecir la presencia de várices esofágicas no es recomendado (46).

Teniendo en cuenta que, de forma independiente, los marcadores no invasivos tienen algunas limitaciones y su uso en la práctica clínica tiene limitaciones, la combinación de diferentes pruebas puede brindar información más precisa; de este modo, se han propuesto modelos matemáticos en los que se integran la elastografía, el recuento de plaquetas y el diámetro esplénico, y en algunas cohortes de pacientes ha permitido seleccionar mejor quiénes requieren estudio endoscópico, lo que disminuye hasta en un $65 \%$ la cantidad de procedimientos invasivos (47).

\section{REFERENCIAS}

1. Berzigotti A, Seijo S, Reverter E, Bosch J. Assessing portal hypertension in liver diseases. Expert Rev Gastroenterol Hepatol. 2013;7(2):141-55. https://doi.org/10.1586/egh.12.83

2. Bosch J, Abraldes JG, Berzigotti A, García-Pagan JC. The clinical use of HVPG measurements in chronic liver disease. Nat Rev Gastroenterol Hepatol. 2009;6(10):573-82. https: / doi.org/10.1038/nrgastro.2009.149

3. Bosch J, García-Pagán JC. Complications of cirrhosis. I. Portal hypertension. J Hepatol. 2000;32(1 Suppl):141-56. https://doi.org/10.1016/s0168-8278(00)80422-5

4. Turco L, Garcia-Tsao G. Portal Hypertension: Pathogenesis and Diagnosis. Clin Liver Dis. 2019;23(4):573-587. https://doi.org/10.1016/j.cld.2019.07.007

5. Tetangco EP, Silva RG, Lerma EV. Portal hypertension: Etiology, evaluation, and management. Dis Mon. 2016;62(12):411-426. https://doi.org/10.1016/j.disamonth.2016.08.001

6. Bosch J. Vascular deterioration in cirrhosis: the big picture. J Clin Gastroenterol. 2007;41 Suppl 3:S247-53. https://doi.org/10.1097/MCG.0b013e3181572357

7. Bloom S, Kemp W, Lubel J. Portal hypertension: pathophysiology, diagnosis and management. Intern Med J. 2015;45(1):16-26. https://doi.org/10.1111/imj.12590

8. García-Pagán JC, Gracia-Sancho J, Bosch J. Functional aspects on the pathophysiology of portal hypertension in cirrhosis. J Hepatol. 2012;57(2):458-61. https://doi.org/10.1016/j.jhep.2012.03.007

9. Garcia-Tsao G, Groszmann RJ, Fisher RL, Conn HO, Atterbury CE, Glickman M. Portal pressure, presence of gastroesophageal varices and variceal bleeding. Hepatology. 1985;5(3):419-24. https://doi.org/10.1002/hep.1840050313

10. Abraldes JG, Villanueva C, Bañares R, Aracil C, Catalina MV, Garci A-Pagán JC, et al. Hepatic venous pressure gradient and prognosis in patients with acute variceal bleeding treated with pharmacologic and endoscopic therapy. J Hepatol. 2008;48(2):229-36. https://doi.org/10.1016/j.jhep.2007.10.008

11. D’Amico G, Luca A. Natural history. Clinicalhaemodynamic correlations. Prediction of the risk of bleeding. Baillieres Clin Gastroenterol. 1997;11(2):243-56. https://doi.org/10.1016/s0950-3528(97)90038-5

12. Bosch J, Abraldes JG, Groszmann R. Current management of portal hypertension. J Hepatol. 2003;38 Suppl 1:S54-68. https://doi.org/10.1016/s0168-8278(02)00430-0

13. Kapoor D, Sarin SK. Pathophysiology of portal hypertension. J Gastroenterol Hepatol. 2002;17 Suppl:S482-7. https://doi.org/10.1046/j.1440-1746.17.s4.14.x

14. Wu Y, Burns RC, Sitzmann JV. Effects of nitric oxide and cyclooxygenase inhibition on splanchnic hemodynamics in portal hypertension. Hepatology. 1993;18(6):1416-21.

15. Schrier RW, Arroyo V, Bernardi M, Epstein M, Henriksen JH, Rodés J. Peripheral arterial vasodilation hypothesis: a proposal for the initiation of renal sodium and water retention in cirrhosis. Hepatology. 1988;8(5):1151-7. https://doi.org/10.1002/hep.1840080532

16. Simonetto DA, Liu M, Kamath PS. Portal Hypertension and Related Complications: Diagnosis and Management. Mayo Clin Proc. 2019;94(4):714-726. https://doi.org/10.1016/j.mayocp.2018.12.020 
17. Lautt WW, Greenway CV. Conceptual review of the hepatic vascular bed. Hepatology. 1987;7(5):952-63. https://doi.org/10.1002/hep.1840070527

18. Pinzani M, Vizzutti F. Reversible factors in portal hypertension. J Gastroenterol Hepatol. 2004;19:S155-S157. https://doi.org/10.1111/j.1440-1746.2004.03668.x

19. de Franchis R, Primignani M. Natural history of portal hypertension in patients with cirrhosis. Clin Liver Dis. 2001;5(3):645-63.

https://doi.org/10.1016/s1089-3261(05)70186-0

20. Westaby S, Wilkinson SP, Warren R, Williams R. Spleen size and portal hypertension in cirrhosis. Digestion. 1978; 17(1):63-8. https://doi.org/10.1159/000198095

21. Abraldes JG, Angermayr B, Bosch J. The management of portal hypertension. Clin Liver Dis. 2005;9(4):685-713, vii. https://doi.org/10.1016/j.cld.2005.08.001

22. Groszmann RJ, Wongcharatrawee $S$. The hepatic venous pressure gradient: anything worth doing should be done right. Hepatology. 2004;39(2):280-2. https://doi.org/10.1002/hep.20062

23. Karagiannakis DS, Voulgaris T, Siakavellas SI, Papatheodoridis GV, Vlachogiannakos J. Evaluation of portal hypertension in the cirrhotic patient: hepatic vein pressure gradient and beyond. Scand J Gastroenterol. 2018;53(10-11):1153-1164. https://doi.org/10.1080/00365521.2018.1506046

24. Moitinho E, Escorsell A, Bandi JC, Salmerón JM, GarcíaPagán JC, Rodés J, et al. Prognostic value of early measurements of portal pressure in acute variceal bleeding. Gastroenterology. 1999;117(3):626-31. https://doi.org/10.1016/s0016-5085(99)70455-5

25. Feu F, García-Pagán JC, Bosch J, Luca A, Terés J, Escorsell $A$, et al. Relation between portal pressure response to pharmacotherapy and risk of recurrent variceal haemorrhage in patients with cirrhosis. Lancet. 1995;346(8982):1056-9. https://doi.org/10.1016/s0140-6736(95)91740-3

26. Escorsell A, Bordas JM, Feu F, García-Pagán JC, Ginès A, Bosch J, et al. Endoscopic assessment of variceal volume and wall tension in cirrhotic patients: effects of pharmacological therapy. Gastroenterology. 1997;113(5):1640-6. https://doi.org/10.1053/gast.1997.v113.pm9352867

27. Idezuki Y. General rules for recording endoscopic findings of esophagogastric varices (1991). Japanese Society for Portal Hypertension. World J Surg. 1995;19(3):420-2; discussion 423. https://doi.org/10.1007/BF00299178

28. Sarin SK, Agarwal SR. Gastric varices and portal hypertensive gastropathy. Clin Liver Dis. 2001;5(3):727-67, x. https://doi.org/10.1016/s1089-3261(05)70190-2

29. De Palma GD, Rega M, Masone S, Persico F, Siciliano S, Patrone F, et al. Mucosal abnormalities of the small bowel in patients with cirrhosis and portal hypertension: a capsule endoscopy study. Gastrointest Endosc. 2005;62(4):529-34. https://doi.org/10.1016/s0016-5107(05)01588-9
30. Shah ND, Baron TH. Endoscopic ultrasound and the liver: current applications and beyond. J Hepatobiliary Pancreat Sci. 2018;25(3):171-180. https://doi.org/10.1002/jhbp.528

31. Schiano TD, Adrain AL, Cassidy MJ, McCray W, Liu JB, Baranowski RJ, et al. Use of high-resolution endoluminal sonography to measure the radius and wall thickness of esophageal varices. Gastrointest Endosc. 1996;44(4):425-8. https://doi.org/10.1016/s0016-5107(96)70093-7

32. Sung JJ, Lee YT, Leong RW. EUS in portal hypertension. Gastrointest Endosc. 2002;56(4 Suppl):S35-43. https://doi.org/10.1016/s0016-5107(02)70084-9

33. Obara K, Irisawa A, Sato Y. Usefulness of EUS in portal hypertension with esophageal varices. Digestive Endoscopy. y 2004;16(Suppl):S168-S172. https://doi.org/10.1111/j.1443-1661.2004.00432.x

34. Qamar AA, Grace ND, Groszmann RJ, Garcia-Tsao G, Bosch J, Burroughs AK, et al. Platelet count is not a predictor of the presence or development of gastroesophageal varices in cirrhosis. Hepatology. 2008;47(1):153-9. https://doi.org/10.1002/hep.21941

35. Wongcharatrawee S, Groszmann RJ. Diagnosing portal hypertension. Baillieres Best Pract Res Clin Gastroenterol. 2000; 14(6):881-94. https://doi.org/10.1053/bega.2000.0136

36. Escorsell A, Garcia-Pagán JC, Bosch J. Assessment of portal hypertension in humans. Clin Liver Dis. 2001;5(3):575-89. https://doi.org/10.1016/s1089-3261(05)70182-3

37. Deng H, Qi X, Guo X. Diagnostic Accuracy of APRI, AAR, FIB-4, FI, King, Lok, Forns, and FibroIndex Scores in Predicting the Presence of Esophageal Varices in Liver Cirrhosis: A Systematic Review and Meta-Analysis. Medicine (Baltimore). 2015;94(42):e1795. https://doi.org/10.1097/MD.0000000000001795

38. Hametner S, Ferlitsch A, Ferlitsch M, Etschmaier A, Schöfl $\mathrm{R}$, Ziachehabi A, et al. The VITRO Score (Von Willebrand Factor Antigen/Thrombocyte Ratio) as a New Marker for Clinically Significant Portal Hypertension in Comparison to Other Non-Invasive Parameters of Fibrosis Including ELF Test. PLoS One. 2016;11(2):e0149230. https://doi.org/10.1371/journal.pone.0149230

39. Qi X, Berzigotti A, Cardenas A, Sarin SK. Emerging non-invasive approaches for diagnosis and monitoring of portal hypertension. Lancet Gastroenterol Hepatol. 2018;3(10):708-719. https://doi.org/10.1016/S2468-1253(18)30232-2

40. Sabbá C, Weltin GG, Cicchetti DV, Ferraioli G, Taylor KJ, Nakamura T, Moriyasu F, Groszmann RJ. Observer variability in echo-Doppler measurements of portal flow in cirrhotic patients and normal volunteers. Gastroenterology. 1990;98(6):1603-11. https://doi.org/10.1016/0016-5085(90)91097-p

41. Kamel IR, Liapi E, Fishman EK. Liver and biliary system: evaluation by multidetector CT. Radiol Clin North Am. 2005;43(6):977-97, vii. https://doi.org/10.1016/j.rcl.2005.07.003 
42. Danrad R, Martin DR. MR imaging of diffuse liver diseases. Magn Reson Imaging Clin N Am. 2005;13(2):277-93, vi. https://doi.org/10.1016/j.mric.2005.03.006

43. Roccarina D, Rosselli M, Genesca J, Tsochatzis EA. Elastography methods for the non-invasive assessment of portal hypertension. Expert Rev Gastroenterol Hepatol. 2018;12(2):155-164. https://doi.org/10.1080/17474124.2017.1374852

44. de Franchis R; Baveno VI Faculty. Expanding consensus in portal hypertension: Report of the Baveno VI Consensus Workshop: Stratifying risk and individualizing care for portal hypertension. J Hepatol. 2015;63(3):743-52. https://doi.org/10.1016/j.jhep.2015.05.022

45. Maurice JB, Brodkin E, Arnold F, Navaratnam A, Paine H, Khawar S, et al. Validation of the Baveno VI criteria to iden- tify low risk cirrhotic patients not requiring endoscopic surveillance for varices. J Hepatol. 2016;65(5):899-905. https://doi.org/10.1016/j.jhep.2016.06.021

46. Colecchia A, Montrone L, Scaioli E, Bacchi-Reggiani ML, Colli A, Casazza G, et al. Measurement of spleen stiffness to evaluate portal hypertension and the presence of esophageal varices in patients with HCV-related cirrhosis. Gastroenterology. 2012;143(3):646-654. https://doi.org/10.1053/j.gastro.2012.05.035

47. Berzigotti A, Seijo S, Arena U, Abraldes JG, Vizzutti F, García-Pagán JC, et al. Elastography, spleen size, and platelet count identify portal hypertension in patients with compensated cirrhosis. Gastroenterology. 2013;144(1):102-111.e1. https://doi.org/10.1053/j.gastro.2012.10.001 